\title{
Reliability Modeling of a Solar Photovoltaic System using Supplementary Variable Technique
}

\author{
Indeewar Kumar \\ Dept. of Maths. and Stats \\ Manipal University Jaipur \\ Jaipur-303007, India
}

\author{
Ashish Kumar \\ Dept. of Maths. and Stats \\ Manipal University Jaipur \\ Jaipur-303007, India
}

\author{
Monika S. Barak \\ Dept. of Maths. and Stats \\ Manipal University Jaipur \\ Jaipur-303007, India
}

\begin{abstract}
In the present study, an attempt has been made to derive the reliability measures of a solar photovoltaic system consisting of four subsystems arranged in a series. There is a single server who visits the system immediately to do repair of the unit. Failure time distributions are negative exponential while the repair time distributions of the subsystems are arbitrary. After repair, subsystems are "as good as new". Moreover, the whole system fails immediately when any subsystem fail. Switch devices are perfect. All random variables are statistically independent. Under these assumptions, using Markov process theory and the Laplace transform, some important reliability indexes and some steady state system indexes are derived. Finally, graphs for various measures of system effectiveness are derived using MATLAB to highlight the importance of the study.
\end{abstract}

\section{Keywords}

Reliability, Availability, Solar Photovoltaic System, Laplace Transformation and Markov Process Theory

\section{INTRODUCTION}

Solar photovoltaic (SPV) produces electrical power from clean, quiet, pollution free sustainable renewable energy source that is accessible whenever there is regular sunshine. SPV systems are recognized as feasible alternatives to energy sources for meeting electric energy demands in remote, isolated un-electrified locations. SPV systems are subject to failure modes: Loose or corroded connections, battery failure, controller failure and module failure represent a few of the things that might go wrong in a SPV system. Limitations in the energy available from SPV system and their intermittent behavior degrade the system reliability.

Many authors including Billinton, R. and Karki, R.[6,7], Billinton, R., Bagen,[9], Cha et al. [8], Jain et al. [4], have contributed in reliability analysis of system including renewable energy sources. Due to the complexity of solar photovoltaic systems the concept of reliability has become a very important factor in the overall system design. While dealing with reliability-based design of machines and structures, we can study the relative importance of mechanical and structural failures of the systems. Reliability analysis of such a system helps us to obtain the necessary information about the control of various parameters. Most of the authors discussed the system possessing Markovian properties. The system having non-Markovian property can be converted into a system having Markovian nature by introducing a new variable called supplementary variable. Initially, Cox [1] used supplementary variable in analysing non-Markovian system and presented a systematic solutions of reliability and availability of that system using supplementary variable technique. Gaver [2] studied a parallel redundant system with constant failure and arbitrary repair rates. Since then several authors have studied the reliability of the various systems using supplementary variable technique. Singh and Dayal [3] used supplementary variable technique for problem formulation. Alfa and Srinivada Rao [5] discussed supplementary variable technique in stochastic models. Ke and Chu [10] studied the comparative analysis of availability for redundant repairable system. Kumar and Saini [11] carried out the profit of a solar Photovoltaic System with Preventive Maintenance. The reliability of solar photovoltaic system has not been discussed so far which plays an important role in the sector of renewal energy. The assumption of constant failure and repair rates may not be practical in any industry.

Keeping this in view, in the present study, we have considered a solar photovoltaic system of four-subsystems subject to constant failure and arbitrary repair rates of the subsystems and discussed the reliability modeling of solar photovoltaic system using supplementary variable technique and Laplace transformation. All repair and failure rates of the subsystems are taken respectively as constant and variable. The unit after repair becomes new. Sufficient repair facilities are provided for repair of the failed units. Under these assumptions, a stochastic model is developed and an attempt has been made to discuss the availability of this system with respect to failure repair rates. To highlight the importance of the study graphs are drawn with MATLAB.

The paper has been organized as follows: the Section 1 is introductory in nature. In Section 2, a brief introduction about system and various notations of the subsystems are presented. The mathematical formulation and solution of the differentialdifference equation of solar photovoltaic system developed using supplementary variable technique, assuming constant failure and variable repair rates presented in Section 3. Certain conclusions drawn from this analysis is also discussed in the Section 4.

\section{SYSTEM DESCRIPTION AND NOTATIONS}

Most of the world power generation systems use large generators and large distribution networks to provide energy to the consumers. In recent years, alternative energy sources, such as photovoltaic, Eolic and biomass have gained ground. The study of photovoltaic energy has shown its technical and economic feasibility as an option to conventional systems. Due to vide applicability of solar systems in energy sector in this paper, we considered solar photovoltaic system consisting of four sub-systems, namely solar cell module, solar charge controller, DC to Ac converter and AC appliances. The complete description of the systems and their notations, required in the mathematical formulation, are as follows: 


\subsection{System Description}

\subsubsection{Solar cell module}

It is a combination of PV plates. Plate consists of photodiodes when light energy incident over the plate the light energy converted into electric energy. Failure of photodiodes causes failure of the system. Overheating also cause damage to the system.

\subsubsection{MPPT}

It is high frequency DC to DC converter. It takes DC input from solar plate and converts it to high frequency $\mathrm{AC}$ voltages and further convert back to high voltage DC voltage. Solar charge controller failure occurs when water humidity and insects cause short circuit which causes failure of the whole system.

\subsubsection{DC to AC converter}

It consists of rectifier circuit, transformer and feedback network. It takes input DC and convert it to desired AC.

\subsubsection{AC Appliances}

$\mathrm{AC}$ appliances cause failure when they are wrongly connected or fail due to individual failure.

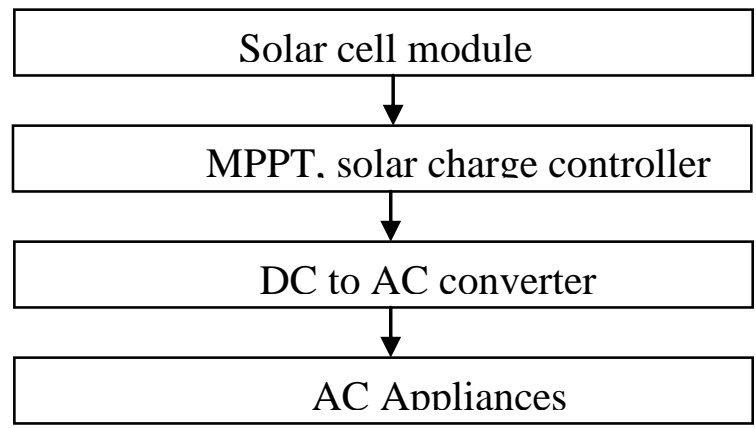

Fig. 1: SPV System Description

\subsection{Notations}

$\alpha_{i} \quad$ Constant failure rate of the subsystems

$(i=1,2,3,4)$

$\beta_{\mathrm{i}}(x)$ Repair rate of the unit and probability density function, for the elapsed repair time ' $x$ '. $(i=1,2,3,4)$

$\begin{array}{ll}S_{\mathrm{i}}(x) & \beta_{\mathrm{i}}(x) e^{\left[-\int_{0}^{x} \beta \mathrm{i}(x) d x\right]} \\ & (\mathrm{i}=1,2,3,4) \\ p_{0}(t) \quad & \begin{array}{l}\text { Definite integral from } 0 \text { to } \mathrm{x} \\ \text { The Probability that at time } \mathrm{t} \text { the }\end{array} \\ & \text { system is in good state }\end{array}$

$p_{i}(x, t) \Delta \quad$ The Probability that at time $\mathrm{t}$ the system is in failed state the elapsed repair time lies in the interval $(\mathrm{x}, \mathrm{x}+\Delta)$

$(\mathrm{i}=1,2,3,4)$

$p(s) \quad$ Laplace transform of $p(t)$

\section{FORMULATION AND SOLUTION OF MATHEMATICAL MODEL}

By probability considerations and continuity arguments, the following difference-differential equations governing the behavior of the system may seem to be good.

$\left(\frac{\partial}{\partial t}+\sum_{i=1}^{4} \alpha_{i}\right) P_{0}(t)=\sum_{i=1}^{4}\left[\int_{0}^{\infty} P_{i}(x, t) \beta_{i}(x) d x\right]$

$\left(\frac{\partial}{\partial t}+\frac{\partial}{\partial x}+\beta_{i}(x)\right) P_{i}(x, t)=0$, where $i=1,2,3,4$

(2)

Boundary Conditions:

$P_{i}(0, t)=\alpha_{i} P_{0}(t)$, where $\quad i=1,2,3,4$

Initial Conditions:

$P_{i}(0)=1 \quad$ when $i=0$

$P_{i}(0)=0$ when $i \neq 0$

Taking LT of equations (1)-(3) and using (4), we get

$$
\begin{aligned}
& \left(s+\sum_{i=1}^{4} \alpha_{i}\right) P_{0}(s)=1+\sum_{i=1}^{4}\left[\int_{0}^{\infty} P_{i}(x, s) \beta_{i}(x) d x\right] \\
& \left(\frac{\partial}{\partial x}+s+\beta_{i}(x)\right) P_{i}(x, s)=0, \text { where } i=1,2,3,4 \\
& P_{i}(0, s)=\alpha_{i} P_{0}(s) \quad \text { where } i=1,2,3,4
\end{aligned}
$$

Integrating equation (6) and further using equation (7), we get

$$
\begin{aligned}
& P_{i}(x, s)=P_{i}(0, s) e_{0}^{\left[-s x-\int_{0}^{x} \beta_{i}(x) d x\right]}, \\
& \text { where } i=1,2,3,4
\end{aligned}
$$

Using equation (8) in equation (5), we get

$$
\begin{aligned}
& \left(s+\sum_{i=1}^{4} \alpha_{i}\right) P_{0}(s)=1+\sum_{i=1}^{4}\left[\alpha_{i} P_{0}(s) S_{i}(s)\right] \\
& {\left[s+\sum_{i=1}^{4} \alpha_{i}\left(1-S_{i}(s)\right)\right] P_{0}(s)=1} \\
& P_{0}(s)=\frac{1}{T(s)}
\end{aligned}
$$

Where

$\mathrm{T}(\mathrm{s})=\left[s+\sum_{i=1}^{4} \alpha_{i}\left(1-S_{i}(s)\right)\right]$

Now, the LT of the probability that the system is in the failed state is given by 
$P_{1}(s)=\int_{0}^{\infty} P_{1}(x, s) d x=\alpha_{1} P_{0}(s) \frac{1-S_{1}(s)}{s}=\alpha_{1} \frac{A_{1}(s)}{T(s)}$

where $A_{1}(s)=\frac{1-S_{1}(s)}{s}$

Similarly

$P_{i}(s)=\int_{0}^{\infty} P_{i}(x, s) d x=\alpha_{i} P_{0}(s) \frac{1-S_{i}(s)}{s}=\alpha_{i} \frac{A_{i}(s)}{T(s)}$

where $A_{i}(s)=\frac{1-S_{i}(s)}{s}$ and $i=2,3,4$

It is worth noting that

$$
\sum_{i=0}^{4} P_{i}(s)=\frac{1}{s}
$$

\section{Evaluation of Laplace transforms of up and down state} probabilities

The Laplace transforms of the probabilities that the system is in up (i.e. good) and down (i.e. failed) state at time " $t$ " are as follows

$P_{u p}(s)=P_{0}(s)=\frac{1}{T(s)}$

$P_{\text {down }}(s)=\sum_{i=1}^{4} P_{i}(s)=\frac{\sum_{i=1}^{4} A_{i}(s)}{T(s)}$

\section{Steady-State Probabilities}

Using Abel's Lemma in Laplace transforms, viz.

$\lim _{s \rightarrow 0} s[Z(s)]=\lim _{n \rightarrow \infty} Z(t)=Z($ say $)$,

Provided the limit on the right hand side exists, the following time independent probabilities have been obtained.

$$
\begin{gathered}
P_{u p}=\frac{1}{\left[1-\sum_{i=1}^{4} \alpha_{i} S_{i}^{\prime}(0)\right]} \\
P_{\text {down }}=\frac{-\sum_{i=1}^{4} \alpha_{i} S_{i}^{\prime}(0)}{\left[1-\sum_{i=1}^{4} \alpha_{i} S_{i}^{\prime}(0)\right]}
\end{gathered}
$$

\section{Reliability indices}

In order to obtain system reliability, consider repair rates (i.e. $\beta_{\mathrm{i}}(x)$ ) equal to zero.Using the method similar to that in section 2 , the differential-difference equations are:

$\left(\frac{\partial}{\partial t}+\sum_{i=1}^{4} \alpha_{i}\right) P_{0}(t)=0$

Theorem 1. The reliability of the system is given by

$$
R(t)=e^{-\left(\sum_{i=1}^{4} \alpha_{i}\right) t}
$$

The proof of the theorem 1 is given in the appendix.

Corollary 1.The mean time to system failure (MTSF) is:

$$
M T S F=\frac{1}{\sum_{i=1}^{4} \alpha_{i}}
$$

Proof. Calculating MTSF $=\int_{0}^{\infty} R(t) d t$ implies the result ' $*$ ' given in the appendix.

\section{Special case}

\section{Availability}

When repair rates follows exponential time distribution Setting $S_{i}(s)=\frac{\beta_{i}}{s+\beta_{i}}$ where $\beta_{i}, \mathrm{i}=1,2,3,4$ are constant repair rates. Putting these values in equation (11), we get

$$
P_{u p}(s)=\frac{\prod_{i=1}^{4}\left(s+\beta_{i}\right)}{s \prod_{i=1}^{4}\left(s+\beta_{i}\right)+\sum_{j=1}^{4}\left[s \alpha_{j} \prod_{i \neq j, i=1}^{4}\left(s+\beta_{i}\right)\right]}
$$

\section{NUMERICAL ANALYSIS}
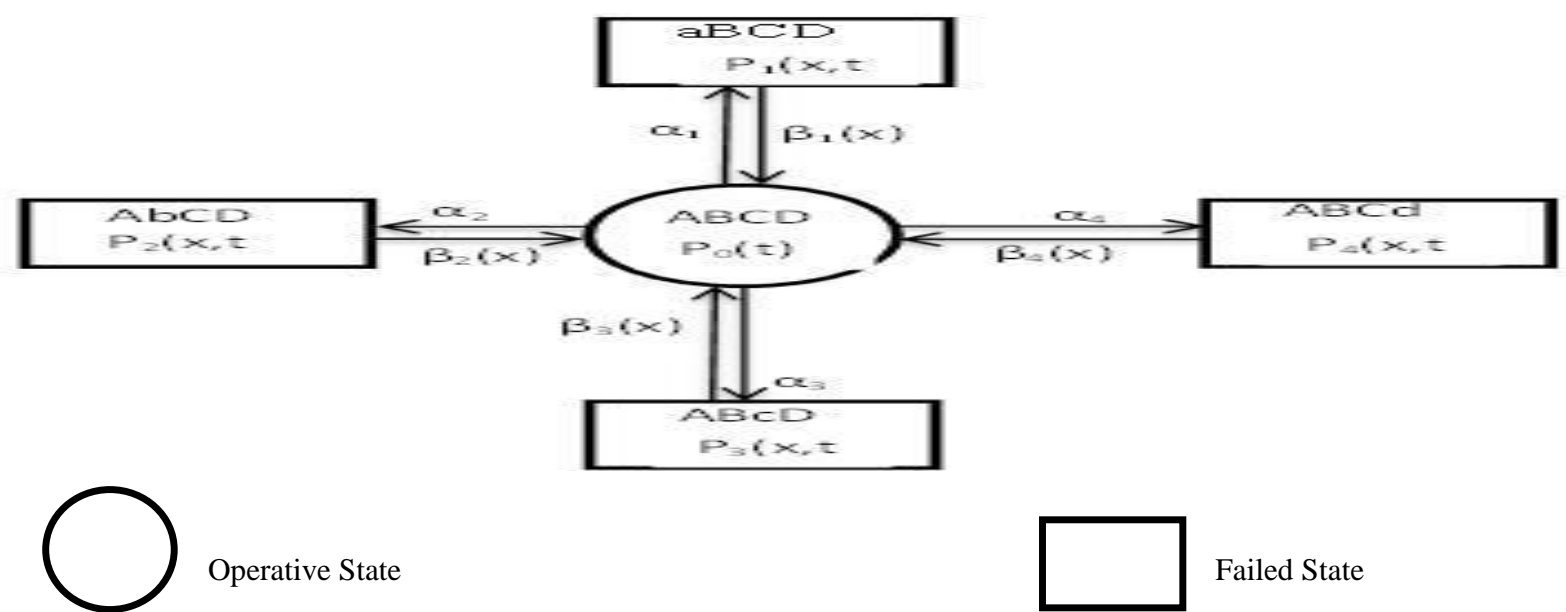

Failed State

Fig. 2: State Transition Diagram 


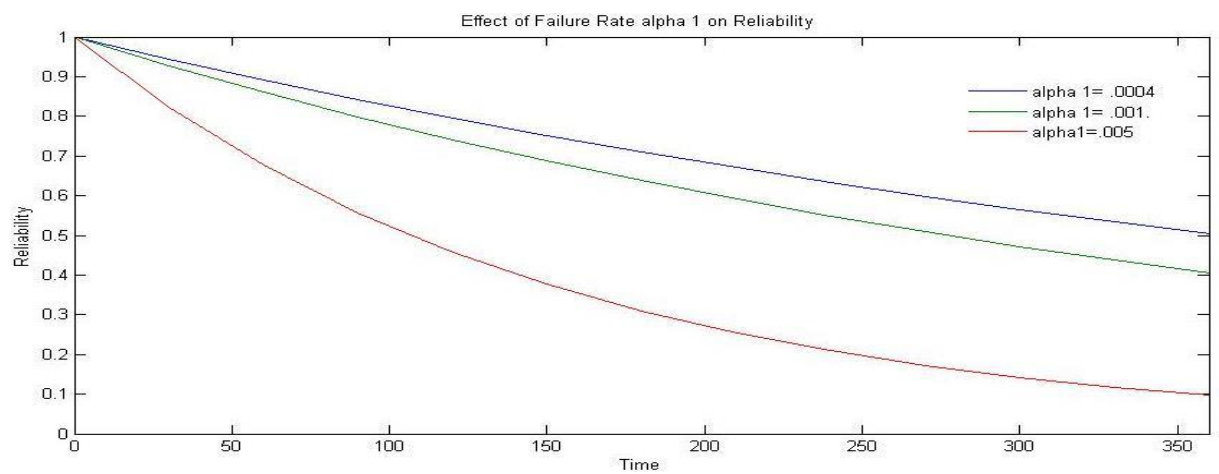

Figure-3: Effect of failure rates $\left({ }^{\alpha_{1}}\right)$ on Reliability $(\mathbf{R}(\mathbf{t}))$

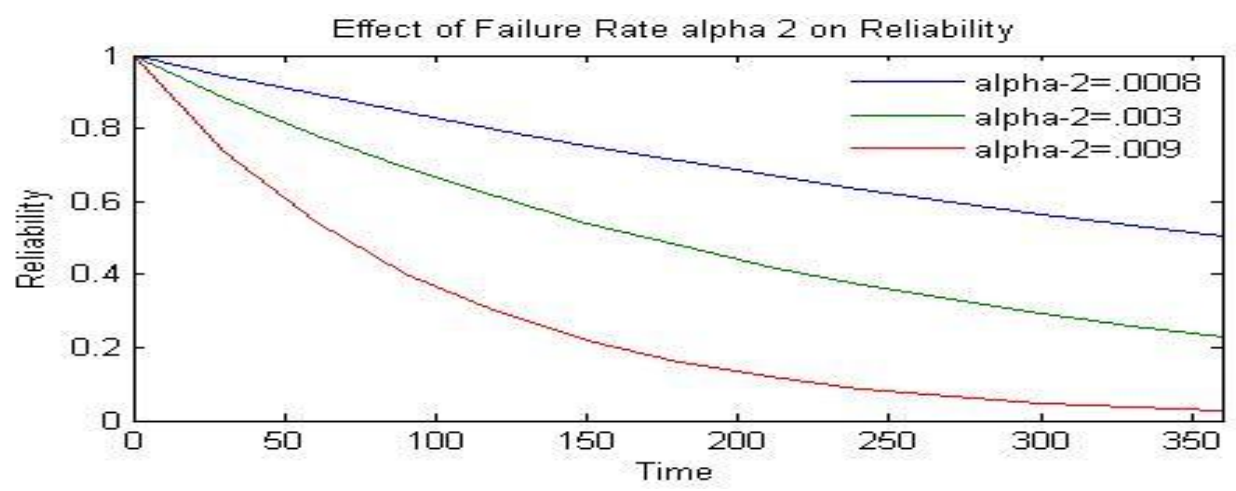

Figure-4: Effect of failure rates $\left({ }^{\alpha_{2}}\right)$ on Reliability $(\mathbf{R}(\mathbf{t}))$

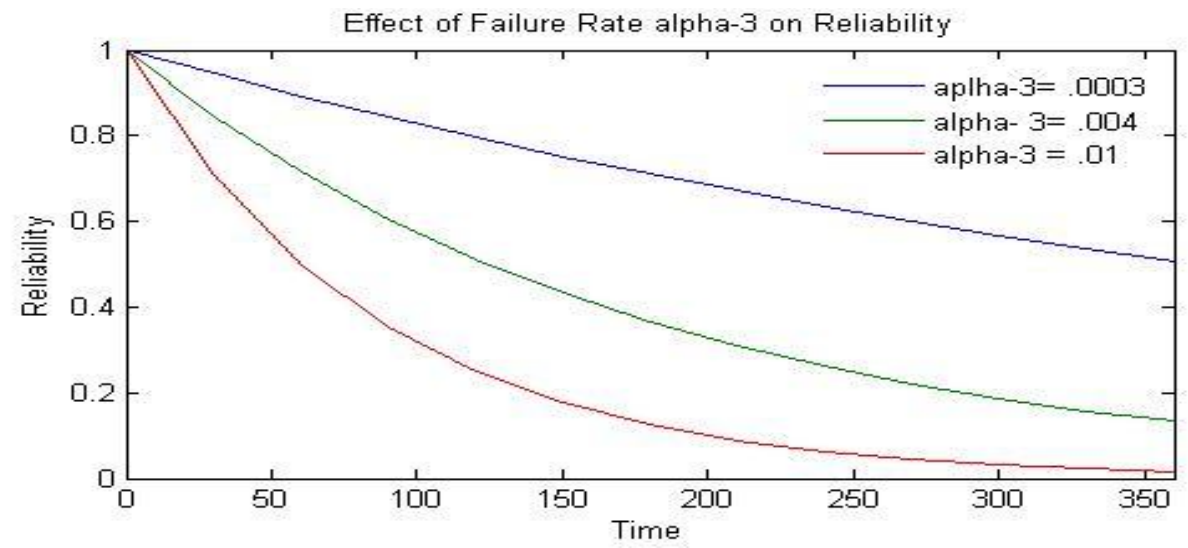

Figure-5: Effect of failure rates ( ${ }^{\alpha_{3}}$ ) on Reliability $(\mathbf{R}(\mathbf{t}))$

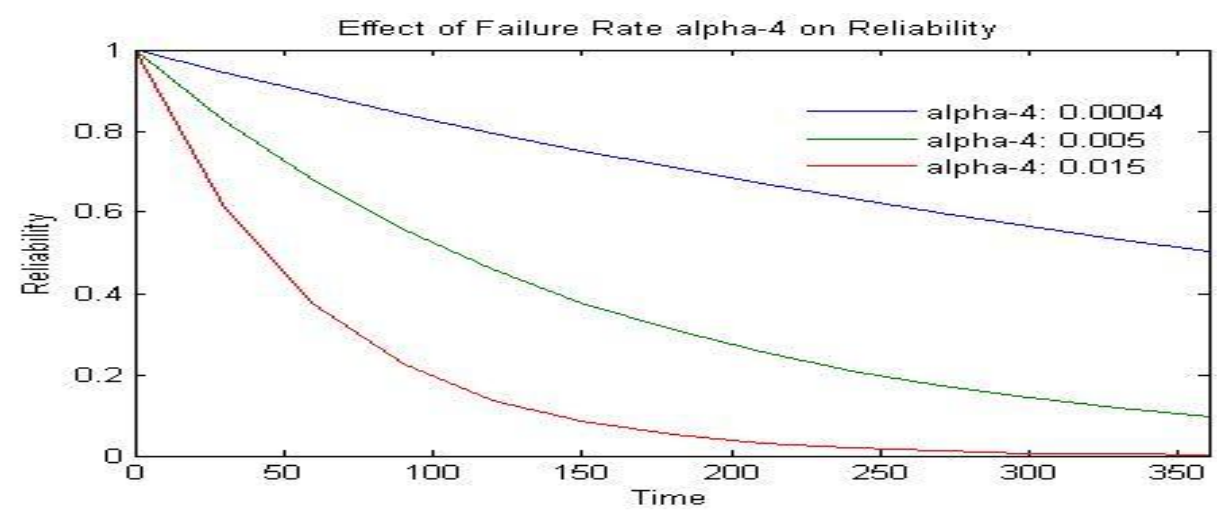

Figure-6: Effect of failure rates ( ${ }^{\alpha_{4}}$ ) on Reliability (R(t) ) 
Table-1: Availability of SPV system w.r.t. failure rate ( $\alpha 1)$ when value of different failure and repair rates are fixed.

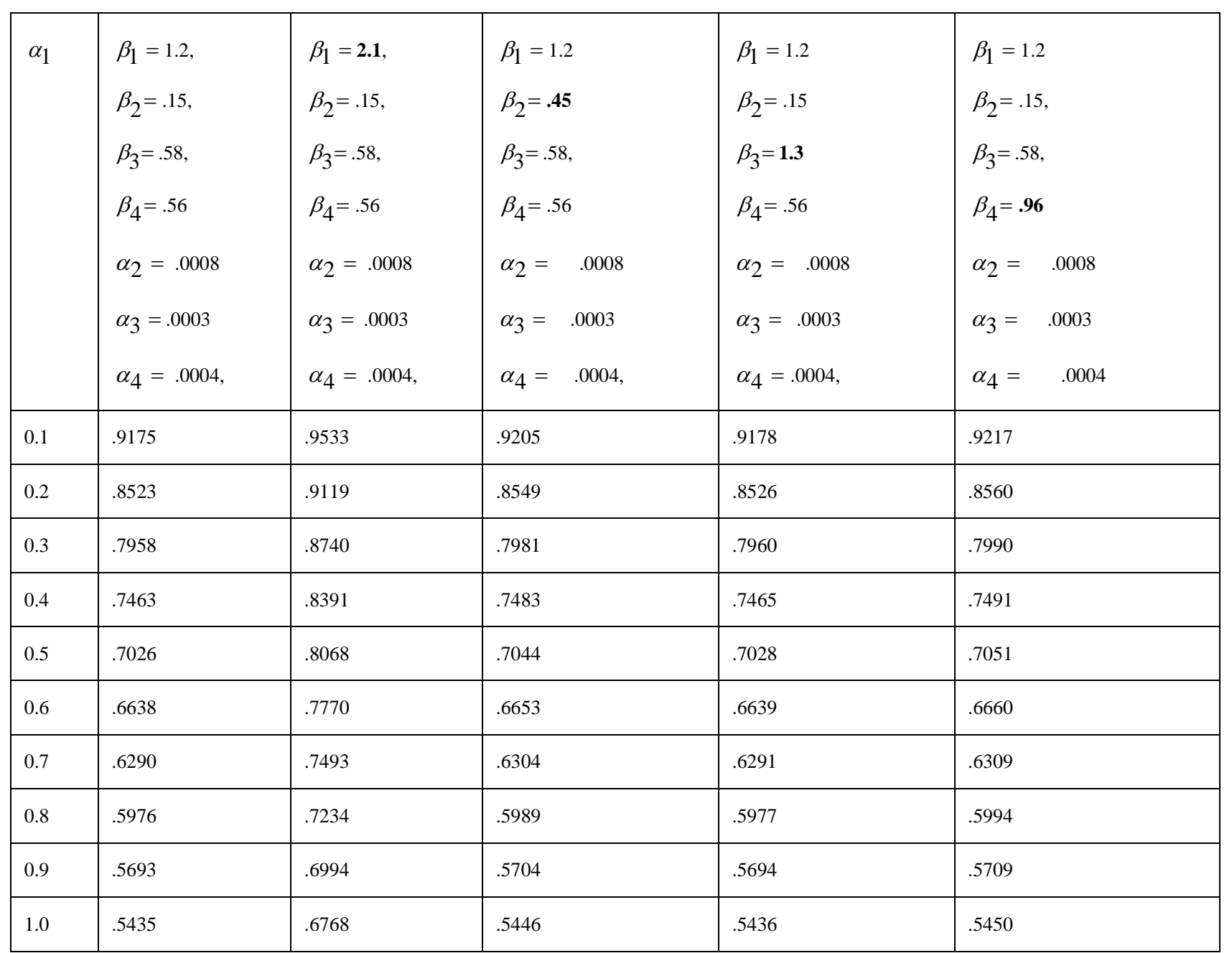

\section{CONCLUSION}

The behavior of system reliability is shown in figures 3- 6 which indicates that reliability of the system decreases with the increase of failure rates $\left(\alpha_{i}\right)$ w.r.t. time and for fixed values of other parameters. Also, it is analyzed that there are sudden jumps in the values of reliability function and over a long period of time the system becomes less and less reliable. Table 1 , shows that availability of the system with respect to failure rate $\left(\alpha_{1}\right)$ and for fixed values of the other parameters. It is revealed that the availability of the system increase with the increase of the repair rates $\left(\beta_{i}\right)$ while availability of the system decrease with the increase of the failure rate $\left(\alpha_{1}\right)$. Thus the availability of the system can be increased by controlling the failure rates and increasing the repair rates of the sub-systems.

\section{Appendix}

Derivation of Equations (1)-(2)

Assuming failure rates of the system are constant and repair rates are variable. By applying supplementary variable technique, we develop the following differential-difference equations associated with the state transition diagram (fig. 1) of the system at time $(t+\Delta t)$ and $(x+\Delta x)$.

$$
\begin{aligned}
& P_{0}(t+\Delta t)=P_{0}(t)\left[1-\sum_{i=1}^{4} \alpha_{i}\right]+\sum_{i=1}^{4}\left[\int_{0}^{\infty} P_{i}(t) \beta_{i}(x) d x \Delta t\right]+o(\Delta t) \\
& P_{i}(t+\Delta t, x+\Delta x)=P_{i}(t, x)\left[1-\beta_{i}(x) \Delta x\right]+o(\Delta t, \Delta x) \\
& \text { where } i=1,2,3,4
\end{aligned}
$$

The proof of theorem 1 is as follows.

Proof: Taking Laplace transform of (18) and using (4) we get

$$
\left(s+\sum_{i=1}^{4} \alpha_{i}\right) P_{0}(s)=1
$$

Using the initial conditions, the solution can be written as

$$
\begin{aligned}
& P_{0}(s)=\frac{1}{s+\sum_{i=1}^{4} \alpha_{i}} \\
& R(s)=\frac{1}{s+\sum_{i=1}^{4} \alpha_{i}}
\end{aligned}
$$

Taking inverse Laplace transform, we get 


$$
R(t)=e^{-\left(\sum_{i=1}^{4} \alpha_{i}\right) t}
$$

\section{REFERENCES}

[1] D.R. Cox, Analysis of Non Markovian stochastic processes by the inclusion of Supplementary variables, Proc. Comb. Phill. Soc. 51 (1955) 433-441.

[2] D.P. Gaver, Time to failure and availability of parallel system with repair, IEEE T. Reliab. R-12 (1963) 30-38.

[3] J. Singh, B. Dayal, A 1-0ut of-N: G system with common cause failure and critical human errors, Microelectron Reliab. 31 (1991) 101-104.

[4] Jain, A., Tripathy, S.C., Balasubramanian, R., 1995. Reliability and economic analysis of a power generation system including a photovoltaic system. Energ. Convers. Manage. 36 (3), 183-189.

[5] A.S. Alfa, T.S.S. Srinivada Rao, Supplementary variable technique in stochastic models, Probab. Eng. Inform. Sci. 14 (2000) 203-218.

[6] Billinton, R., Karki, R., 2001. Maintaining supply reliability of small isolated power systems using renewable energy. IET Proc. Gener. Transm. Distrib. 148 (6), 530-534

[7] Billinton, R., Karki, R., 2003. Reliability/cost implications of utilizing photovoltaics in small isolated power systems. Reliab. Eng. Syst. Saf. 79, 11-16.

[8] Cha, S.T., Jeon, D.H., Bae, I.S., Lee, R., Kim, J.O., 2004. Reliability evaluation of distribution system connected photovoltaic generation considering weather effects. Proceedings of 8th International Conference on Probabilistic Methods Applied to Power System. Iowa State University, Ames, Iowa, pp. 451-456.

[9] Billinton, R., Bagen, 2006. Generating capacity adequacy evaluation of small stand alone power system containing solar energy. Reliab. Eng. Syst. Saf. 91, 438-443.

[10] J.C. Ke, Y.K. Chu, The comparative analysis of availability for redundant repairable system, Appl. Math. Comput. 188 (2007) 332-338.

[11] Kumar, A. and Saini, M. Profit Analysis of Solar Photovoltaic System with Preventive Maintenance, Int. J. Modern Math. Sci. 2014, 10(3): 247-259. 